\title{
Prediction of municipal solid waste generation for developing countries in temporal scale: A fuzzy inference system approach
}

\author{
Kolekar K.A., Hazra T. ${ }^{*}$ and Chakrabarty S.N. \\ Environmental Engineering division, Department of Civil Engineering, Jadavpur University, 188 Raja S. C. Mallick Road, Jadavpur, Kolkata \\ -700032 , India
}

Received: 20/04/2017, Accepted: 20/08/2017, Available online: 23/10/2017

*to whom all correspondence should be addressed: e-mail: tumpa_hazra@yahoo.com

\begin{abstract}
Fuzzy Inference System (FIS) based prediction models for the Municipal Solid Waste (MSW) generation has been developed in the present work to study the influences of total population, percapita annual income, literacy rate, age group and monthly consumer expenditure on temporal variability of MSW generation for Kolhapur city, India. Ten models were developed considering two input variables at a time to study the effect of the socioeconomic and demographic parameters on MSW generation. Finally, all five input variables were considered in a single model to predict MSW generation in a temporal scale. Result shows that, the model with input variables consumer expenditure and age group was best fitted with highest coefficient of determination (0.985) value and lowest standard error of the estimate (1.562) value for the modelling period. For the design period, models related to consumer expenditure show higher waste generation. Models related to population and age show prediction similar to 'Kolhapur Municipal Corporations' prediction. However model with input literacy and income shows very low waste generation prediction. The proposed modelling technique is very useful in MSW generation prediction for a temporal scale in uncertain and random environment globally.
\end{abstract}

Keywords: Municipal solid waste, Fuzzy inference system, Generation prediction, Temporal scale, Statistical analysis

\section{Introduction}

The continuous increasing rate of population, changing lifestyles along with consumption pattern due to economic development creates complexity in both quantity and quality of MSW, especially in developing countries. Throughout the world, the management of MSW has been creating a crucial issue in every municipality. Municipal Solid Waste Management (MSWM) system in developed countries like US, Japan and Western Europe are well controlled than in developing countries (Zia and Devadas, 2008). MSWM mainly depends upon the accurate quantification and prediction of MSW generation, since it is the foundation and prerequisite for proper MSWM planning. However, to develop efficient MSWM system through a process of continual improvement, it will not be sufficient enough to know the total quantity of wastes being generated in a region; both-the quantity and temporal distribution of waste must be described as well.

MSW generation prediction is a very complex task since it depends on many attributes both in qualitative and quantitative terms, the particularities and constraints of different areas under consideration as well as the errors in the estimation of factors. The attributes affecting MSW generation changes with local and global scale. In actual practices, due to uncertainties and unavailability of historical records regarding temporal MSW generation for long term and factors affecting generation for developing countries mainly for budget and manpower constraints, data-driven modeling methods are needed for prediction of MSW generation. It has been noticed that the physical and chemical components of MSW depends upon a number of variables such as age, literacy, income and consumption pattern where total MSW generation depends on total population. Conventional methods for predicting waste generation are weight volume analysis, material balance analysis and load-count analysis (Tchobanoglous et al., 1993). Load count analysis and weight volume analysis are based on the waste collection and these cannot depict the rate of waste generation. On the other hand for successful application of material balance analysis, accurate records of all input and output activities are required along with a well-defined boundary. For developing countries, it is a very complex task.

At the same time, the data regarding attributes affecting MSW generation are recorded in the very imprecise way and linguistic terms are used to describe the attributes. Classic statistical models, such as regression models, cannot be useful for handling linguistic data and their precision is poor when inaccurate data are used. At the same time, it cannot handle all the attributes affecting MSW generation (Noori et al., 2009). These methods determine MSW generation based on the coefficient of per capita basis. Since the coefficient changes with time, these methods become useless and needs advance techniques for predicting in the dynamic and nonlinear system. Several researchers have found time series models are very effective for MSW generation prediction considering seasonal impacts. The time series models require a large 
number of data for generation prediction even for short term period also (Intharathirat et al., 2015). In case of input-output analysis and system dynamics models, a causal relationship between influencing attributes and MSW generation is required to be established, requires huge significant continuous data.

In various environmental problems, artificial intelligence models like fuzzy logic and artificial neural network (ANN) have also been fruitfully applied (Gemitzi et al., 2011). In artificial intelligence models, the relation between input and output variables are first established from previous experiences and then future outputs will be anticipated (Abbasi et al., 2014). Without the need of complete discernment of MSW generation process, these datadriven models have high ability to model waste generation fluctuations in temporal scale. Of the artificial intelligence models, ANN models have been found to predict MSW generation more accurately than conventional regression analysis and time series analysis (Inthariat et al., 2015). ANN model also requires large historical data along with the problems of over-fitting in training of the models, difficulty in the determination of network architecture (Abbasi et al., 2014). Similarly, it cannot handle linguistic data.

Considering modeling methods that have been used so far, fuzzy logic has been preferred as a decision-making tool for the complex situation since it allows treating uncertain, qualitative and contradictory data in some extent. Whereas, particularly for developing countries, the data related to MSW generation are associated with some errors which make the fuzzy logic approach most suitable. It is more in line with human thought since it basically relates input variables with output variables by some possible rules. Decision makers can generate their own set of rules to test the fitness of the rule for the fuzzy model. This makes the fuzzy logic approach more user-friendly even in a complex situation. Fuzzy logic based modeling method has been successfully applied for MSW generation prediction since 2000. Karadimas et al., (2006) and Oumarou et al., (2012) have predicted MSW generation using residential activity and commercial activity such as real estate commercial value, maximum building density factor, electricity bill, commercial traffic, land use and building area. Lozano-Olvera et al., (2008) considered family members, education, income and consumption characteristics of the household as input variables for predicting packaging waste profile and its quantity successfully. For prioritizing factors influencing to waste generation, Khan and Farooqi (2012) used fuzzy analytic hierarchy process.

All the researchers predicted waste for a specific year and the models are very case specific, whereas to design a MSWM facility, it is required to predict waste quantity for a design period and also to study the effects of influencing variables so that it may be a global model.

The main aim of this paper is to develop a Fuzzy Inference System (FIS) based simple global model to predict Municipal Soild Waste (MSW) generation for a design period to select sustainable landfill site for disposal of MSW and to observe the effects of socio-economic and demographic variables on waste generation. By using this method one can easily identify the influencing independent variable on MSW generation. The applicability of the developed model was shown using real data of socioeconomic and demographic conditions of Kolhapur city, India.

\section{Overview of the study area}

Kolhapur city lies between latitude $16^{\circ} 42^{\prime}$ North and longitude $74^{\circ} 14^{\prime}$ East is considered as the study area. Kolhapur is a district place of Maharashtra state, India. It is the door of Konkan. From ancient time, this city has been known as Dakshin Kashi and lots of pilgrims visit this place every year. Kolhapur is also famous for wrestling and Kolhapuri Chappals (slippers). For collection of MSW, Kolhapur city is divided into 5 zones and 11 wards. MSW was collected by private organization till Feb 2012. Due to poor performances in MSW collection by the private organization, that practice was discontinued. Kolhapur municipality itself is now collecting MSW. Moreover, for scientific processing on Built Own Operate and Transfer (BOOT) basis, Kolhapur Municipal Corporation (KMC) has appointed Zoom Bio Fertilizer Pvt. Ltd, Mumbai in the year 2000 for 30 years. Unfortunately, since $15^{\text {th }}$ June 2011, this company has failed to process MSW. At present, there is no sanitary landfill site for Kolhapur and all MSW are dumped at waste processing site within the city area as open dumping. To select the sanitary landfill site, accurate MSW generation prediction is essential. Therefore, FIS based models have been developed to predict MSW generation.

\section{Methodology}

The steps that have been followed in this study are

- Identifying and selecting the independent variables

- Collecting and analysing the data related the input and output variables

- Developing the models

- Validating the model and verifying the model performances by calculating statistical indices

- Studying the effects of various independent variables on MSW generation

- Predicting MSW generation for a temporal scale

3.1. Identification and selection of the independent variables for MSW generation prediction

In model development, selection of independent variables plays a crucial role. These variables were identified based on critical literature review. The independent variables may be classified mainly as socio-economic conditionrelated and demographic condition-related. Socioeconomic condition-related variables are income, gross domestic product (GDP), expenditure, tax, employment, unemployment, retail sales and material consumption, energy consumption, gas consumption, power demand etc. and these variables affect MSW generation for both developed countries and developing countries. Of the 
socio-economic condition related independent variables, income is the most widely and significantly hypothesized attribute in MSW generation prediction mainly for developing countries. For this reason, per capita annual income was used in the present study. Since a significant portion of the income is used for savings and paying debts therefore monthly per capita consumer expenditure was also considered for model design. On the other hand, total population, age and literacy are the most commonly used demographic attributes affecting MSW generation for both developed countries and developing countries. The total population is most widely and significantly used variables affecting MSW generation followed by age and literacy.
Since the primary objective of the present study is the selection of landfill site hence total MSW generation prediction is required. Therefore total population was considered as input variable along with age group and literacy rate. Detailed descriptions of selections of input variables were presented in Kolekar et al., (2016). Finally most commonly used five input variables were short listed (total population, per capita annual income, literacy rate, age group and monthly per capita consumer expenditure) for predicting MSW generation. The selected variables were obtained significantly correlated [0.01 level (2-tailed)] with each other and MSW generation data provided by the $\mathrm{KMC}$ in Pearson correlations test as presented in Table1.

Table1. Pearson correlation between variables and output for modeling period

\begin{tabular}{|c|c|c|c|c|c|c|c|c|c|c|}
\hline & & \multirow[b]{2}{*}{ POP } & \multicolumn{4}{|c|}{ AGE } & \multirow[b]{2}{*}{ EXP } & \multirow[b]{2}{*}{ LIT } & \multirow[b]{2}{*}{ INC } & \multirow[b]{2}{*}{ MSW } \\
\hline & & & 0 to 4 & 5 to 14 & 15 to 59 & $\begin{array}{l}60 \text { and } \\
\text { above }\end{array}$ & & & & \\
\hline & POP & 1 & & & & & & & & \\
\hline \multirow{4}{*}{ AGE } & 0 to 4 & $-.989^{* *}$ & 1 & & & & & & & \\
\hline & 5 to 14 & $-.997^{* *}$ & $.995^{* *}$ & 1 & & & & & & \\
\hline & 15 to 59 & $.996^{* *}$ & $-.998^{* *}$ & $-1.000^{* *}$ & 1 & & & & & \\
\hline & $60 \&$ above & $.997^{* *}$ & $-.995^{* *}$ & $-1.000^{* *}$ & $1.000^{* *}$ & 1 & & & & \\
\hline & EXP & $.998^{* *}$ & $-.988^{* *}$ & $-.998^{* *}$ & $.996^{* *}$ & $.998^{* *}$ & 1 & & & \\
\hline & LIT & $.993^{* *}$ & $-.999^{* *}$ & $-.998^{* *}$ & $1.000^{* *}$ & $.998^{* *}$ & $.993^{* *}$ & 1 & & \\
\hline & INC & $.994^{* *}$ & $-.999^{* *}$ & $-.999^{* *}$ & $1.000^{* *}$ & $.999^{* *}$ & $.994^{* *}$ & $1.000^{* *}$ & 1 & \\
\hline & MSW & $.994^{* *}$ & $-.998^{* *}$ & $-.997^{* *}$ & $.999^{* *}$ & $.998^{* *}$ & $.993^{* *}$ & $.999^{* *}$ & $.999^{* *}$ & 1 \\
\hline
\end{tabular}

**. Correlation is significant at the 0.01 level (2-tailed). POP-Total population, AGE- Age group, EXP-Monthly per capita consumer expenditure, LIT-Literacy rate, INC-Per capita annual income, MSW-Municipal solid waste generated

\subsection{Collection and analysis of data}

Incomplete database and presence of uncertainty within considered variables has created complexity in waste generation prediction. Unlike developed countries, in developing countries like India, there is a lack of available continuous historical records. Therefore, for the development of FIS based model for the study area, authors relied on the historical data of population for Kolhapur City presented in the Environmental Status Report (ESR) 2012-13 for the period of 1901 to 2011 (Kolhapur Municipal corporation, 2013). Data related to age group in percentage were retrieved from the national sample survey report (Directorate of Economics and Statistics Maharashtra, 2012). Secondary data related other independent variables were collected from the Statistical Department. Data related to per capita annual income and literacy rate (District Socio-Economic Review of Kolhapur, 2013) were considered for the whole Kolhapur district, where monthly per capita consumer expenditure (Directorate of Economics and Statistics Maharashtra, 2015) was taken into consideration for Maharashtra state urban area only. The amount of waste reported by KMC, estimated from the vehicles capacity and/or weight at the dump sites were used for model development. Modeling period for the waste generation prediction was considered 10 years (2004-2013) while for the waste generation prediction for the selection of the sanitary landfill site, the design period was considered 26 years (2014-2040). Due to lack of data, data regarding all input variables were extended upto 2040 by trend extension based on the best coefficient of determination $\left(R^{2}\right)$ value.

\subsection{Development of model}

In this research work, first 10 models were developed considering two separate input variables (like total population-literacy rate) at a time to study the effects of input variables on MSW generation using Fuzzy Logic Tool Box of MATLAB $^{\oplus}$ (R2013b). Finally, a single model was developed considering all the five input variables (total population, per capita annual income, literacy rate, age group and monthly per capita consumer expenditure) to predict the MSW generation for the modeling period (2004-2013). After validation, the developed models were used for future long-term MSW generation prediction for the design period (2014-2040). Figure1 represents the schematic diagram of the steps of the model which are described below in details.

\subsubsection{Fuzzification}

After collecting data regarding input and output variables, all data were transformed into degrees of belonging to the appropriate fuzzy sets, by assigning membership functions based on intuition and literature review. Triangular and trapezoidal membership functions were applied to describe the attributes. The membership functions that were assigned to each variable are described below.

\section{i) Total population}

Indian census defined, a place, with a population of 0.1 million, as a town and population above 0.1 million as a city. Above 1 million populations, cities are called million plus cities or urban agglomeration (Arthapedia, 2013). Population 0.05 to 0.1 million was considered as very low 
and population 0.1 to 1 million was divided into four groups as low, low medium, medium and high for model development purpose as presented in Figure 2a. Total Population was considered in lakh (1lakh $=0.1$ million) for defining the membership functions in the present work.

ii) Per capita annual Income

In India, households with annual family income less than $\$ 2992.39$ ( $\$ 1=₹ 66.84)$ are considered as a low-income group, household having annual income with $\$ 2992.22$ to $\$ 14961.93$ as medium income group and those having annual income above $\$ 14961.93$ as a high- income group (Gyan research and analytics, 2012). Since total population was taken as an input variable hence per capita annual income instead of household annual income was considered. For per capita annual income calculation, household size was assumed as four. When annual family income is exactly $\$ 2992.39$ i.e. per capita $\$ 748.09$, in Boolean logic it comes under medium income group. In reality, it may not be feasible, since decreasing \$1income only causes per capita family income suddenly to be under low income group. So, the low-medium group was assigned in between low and medium income group. Therefore, annual per capita income in thousand $₹$ was divided into four groups as low, low medium, medium and high for model development as shown in Figure2b.

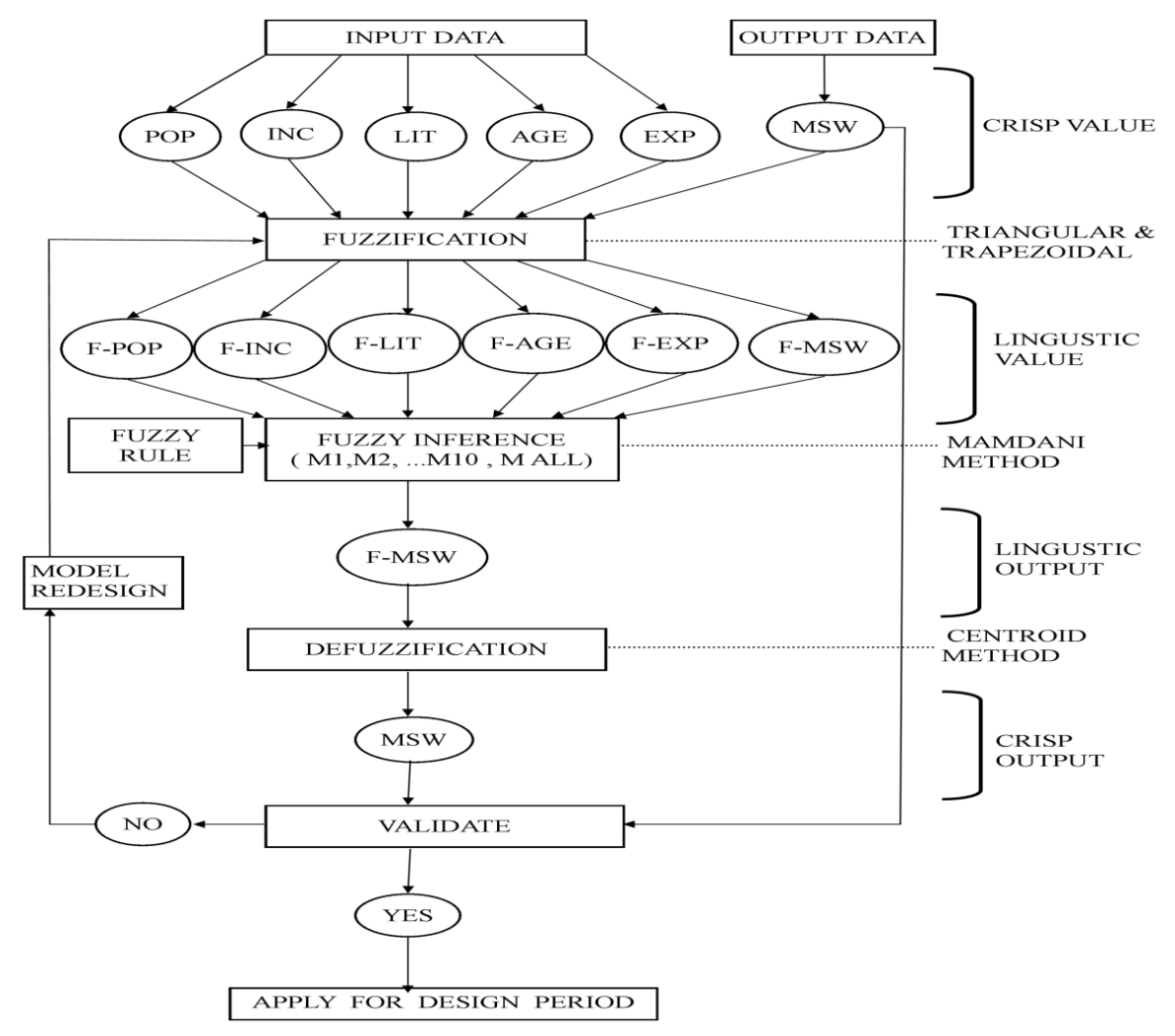

Figure1. Flow diagram of model development: POP-Total population, INC-Per capita annual income, LIT-Literacy rate, AGE- Age group, EXP-Monthly per capita consumer expenditure, MSW-Municipal solid waste generation, F... -F indicates fuzzified, M...- M indicates model name

\section{iii) Literacy rate}

Literacy rate of the total population was classified broadly into three categories as Low (0 to 30\%), Medium (31-70\%) and High (71-100\%) (Planning commission of India, 2001). For the model development, the literacy rate was divided into six categories as very low, low, medium, high medium, high and very high as shown in Figure2c.

\section{iv) Age group}

Directorate of Economics and Statistics, Maharashtra (2012) report has classified the percentages of the population of the urban area of Maharashtra into four age groups as 0 to 4,5 to 14,15 to 59 and 60 and above. For the model development, each age group was considered individually and further classified as low, medium and high based on the available data range as shown in Figure $2 d$, e, $f, g$. The main purpose of considering individual group was to identify the effect of each group on MSW generation.

v) Monthly per capita consumer expenditure

Consumer expenditures are strongly administered by consumer's necessities, behaviours, cultural practices, market trends, availabilities and preferences. Wide ranges of variation have been observed in the database regarding monthly per capita consumer expenditure (in thousand ₹ ) and there are no standard classification rules available in the literature. Hence, this entire database was classified into four groups to assign membership function as very low, low, medium and high as per convenience for model development as shown in Figure $2 \mathrm{~h}$.

vi) Daily MSW generation 
Available data regarding MSW generation reveals that daily MSW generation for KMC area lies between 130 to 200 $\mathrm{t} \mathrm{d}^{-1}$. Hence, waste generation was classified into very low, low, low medium, medium, high medium, low high, high and very high to predict future MSW generation for both modelling and design period and membership functions were assigned according to the data range as presented in Figure2i.

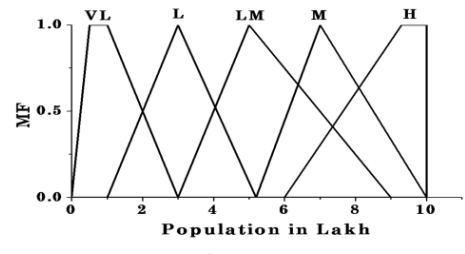

a

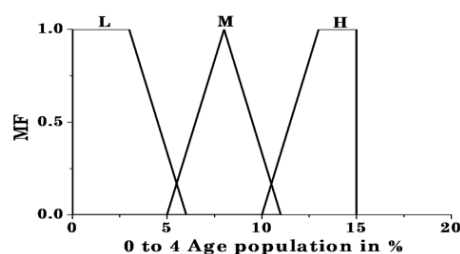

d
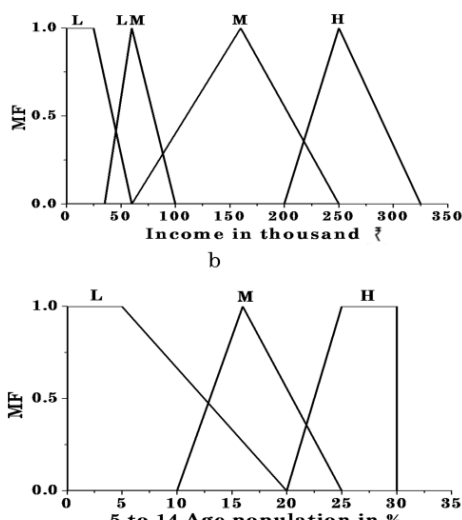
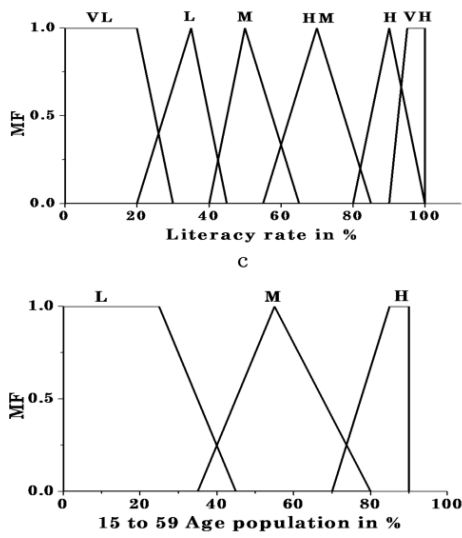

$\mathrm{f}$

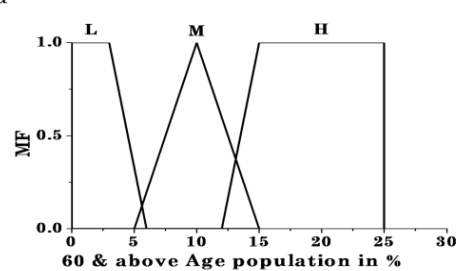

$\mathrm{g}$

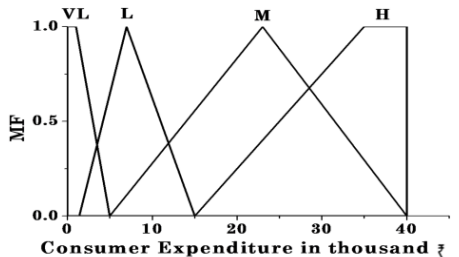

$\mathrm{h}$

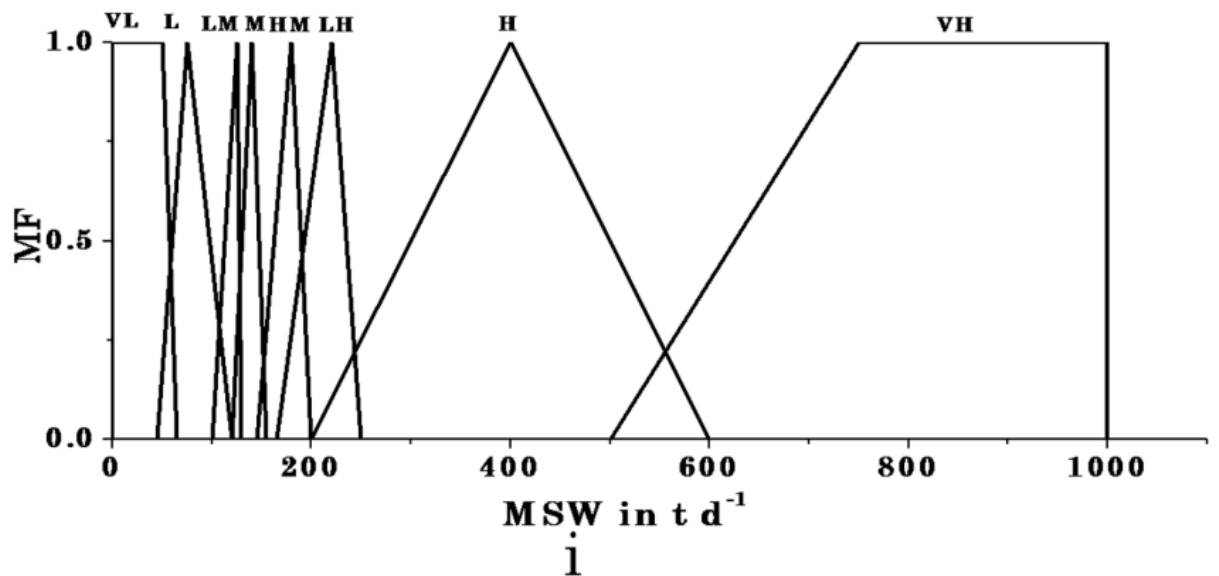

Figure2. Membership functions for variables:(a) Total population (b) Per capita income (c) Literacy rate(d) 0 to 4 age group (e) 5 to 14 age group (f) 15 to 59 age group (g) 60 and above age group (h) monthly per capita consumer expenditure (i) Waste generation

VL-Very low, L-Low, LM-Low medium, M-Medium, HM-High medium, LH-Low high, H-High, VH-Very high, MF-Degree of membership, 1 lakh = 0.1 million

\subsubsection{Fuzzy rules}

During model development, the relationships amongst the input and output variables were formulated by 'If-Then' fuzzy rules using 'AND' operator. Equal weights were assigned to each rule. Details of models and associated rules have been presented in Table 2 along with their short name. For the two input variable models, number of rules were calculated by using formula $m_{i} \times n_{j}$ (where $m$ is the number of levels of $i^{\text {th }}$ variable and $n$ is the number of levels of $\mathrm{j}^{\text {th }}$ variable). For all input variable model, the rules were decided based on the formula $\prod_{i=1}^{n} m_{i}$ where $m_{i}$ is the number of levels of the $i^{\text {th }}$ variable. Since five input variables with their levels would generate 38880 numbers of rules which is quite impossible to handle even with software. Hence only applicable rules based on intuition and literature were considered during model development. With the help of software inbuilt fuzzy inference rule editor a set of 'If-Then' rules were formulated based on the training data to achieve the desired output. These rules were further modified based on the testing database. 


\subsubsection{Fuzzy inference}

In this step, the truth of the consequent (after then) is inferred from the degree of truth of antecedent (after If). A fuzzy inference has provided a basis from which decisions can be made. Mamdani modeling method with minimization implication was applied here for the prediction of MSW generation.

\subsubsection{Defuzzification}

The outputs obtained from the model were also in the form of a fuzzy set. For the purpose of the converting these outputs into single crisp value, defuzzification process was required. There are five built-in defuzzification methods available, of which centroid method is the most popular hence was applied in this prediction process.

Table2. Details of models

\begin{tabular}{clc}
\hline $\begin{array}{c}\text { Model } \\
\text { Name }\end{array}$ & \multicolumn{1}{c}{ Inputs considered } & Number of rules \\
\hline M1 & Total population and literacy rate (POP-LIT) & 30 \\
\hline M2 & Total population and monthly per capita consumer expenditure (POP-EXP) & 20 \\
\hline M3 & Total population and per capita annual income (POP-INC) & 20 \\
\hline M4 & Total population and age group (POP-AGE) & 405 \\
\hline M5 & Monthly per capita consumer expenditure and literacy rate (EXP-LIT) & 24 \\
\hline M6 & Monthly per capita consumer expenditure and per capita annual income (EXP-INC) & 16 \\
\hline M7 & Monthly per capita consumer expenditure and age group (EXP-AGE) & 324 \\
\hline M8 & Age group and literacy rate (AGE-LIT) & 486 \\
\hline M9 & Age group and per capita annual income (AGE-INC) & 324 \\
\hline M10 & Literacy rate and per capita annual income (LIT-INC) & 24 \\
\hline M all & All five variables in a single model (M all) & 1536 (applicable) \\
\hline Number of rules: e.g. total population mi $m_{i}=5$ literacy rate $n_{j}=6$, therefore number of rules= 5×6=30. POP-Total population, AGE- Age group, EXP- \\
\hline
\end{tabular}

\subsection{Validation of the model}

The crisp value regarding MSW generation obtained from each model was compared with the actual MSW generation data obtained from the $\mathrm{KMC}$ in training and testing process and rules applied for each model were modified accordingly. Then the models were validated with the remaining MSW generation data. Of the data of the modeling period (2004-2013) related to the input variables obtained from the best-fitted curves, randomly selected $40 \%$ data were used for training, 30\% for testing and remaining $30 \%$ for validation of the models. After validation, the developed models were used for future long-term MSW generation prediction for the design period of 2014 to 2040.

\section{Results and discussion}

The results obtained from the developed models were compared with the actual MSW generation data for the modeling period (2004 to 2013) as presented in Figure3.

To evaluate the performance of the models, model results were assessed with two descriptive statistics such as determination coefficient $\left(R^{2}\right)$ and standard error of the estimate using SPSS (20) as shown in Table 3.

From Table 3, it is evident that all models reveal the high performance of predicting MSW generation with excellent accuracy since the coefficient of determination $\left(R^{2}\right)$ values range from 0.924 to 0.985 . As per $R^{2}$ and standard error of the estimate values, the best-fitted model for the modeling period is "M7" model (i.e. monthly per capita consumer expenditure and age group as input variables).

Finally, MSW generation predicted by these models were compared with MSW generation predicted by the KMC
(Kolhapur Municipal Corporation, 2008) for the design period (2014-2040).

From Figure4, it is revealed that KMC predicted nearly $400 \mathrm{t} \mathrm{d}^{-1} \mathrm{MSW}$ generation in the year 2040. The models considering monthly per capita consumer expenditure as an input variable (M5: monthly per capita consumer expenditure and literacy rate; M6: monthly per capita consumer expenditure and per capita annual income and M7: monthly per capita consumer expenditure and age group) predicted higher MSW generation than KMC predicted for the design period. While, Models "M1" (total population and literacy rate), "M2" (total population and monthly per capita consumer expenditure), "M3" (total population and per capita annual income), "M4" (total population and age group), "M8" (age group and literacy rate) and "M9" (age group and per capita annual income) predicted waste generation in the range of 350 to $400 \mathrm{t} \mathrm{d}^{-1}$ which is similar to KMC's prediction in connection with MSW generation. The models considering total population as input variables (M1, M2, M3 and M4) showed similar MSW generation trend for the design period, which could be hypothesized that total population is the significant governing attribute for MSW generation (Zhen-Shan et al., 2009; Younes et al., 2016).

On the other hand, no such significant variation in MSW generation was observed for model considering Literacy rate and percapita annual income as input variables (M10) for the design period. Models considering per capita annual income as input variable revealed that per capita does not hypothesize significantly as a governing attribute of MSW generation. From the data analysis, it is obvious that Literacy rate for KMC area will be reached to $100 \%$ within the year 2020. Therefore model considering per capita 
annual income and literacy rate did not show any variation

in MSW generation for the design period.

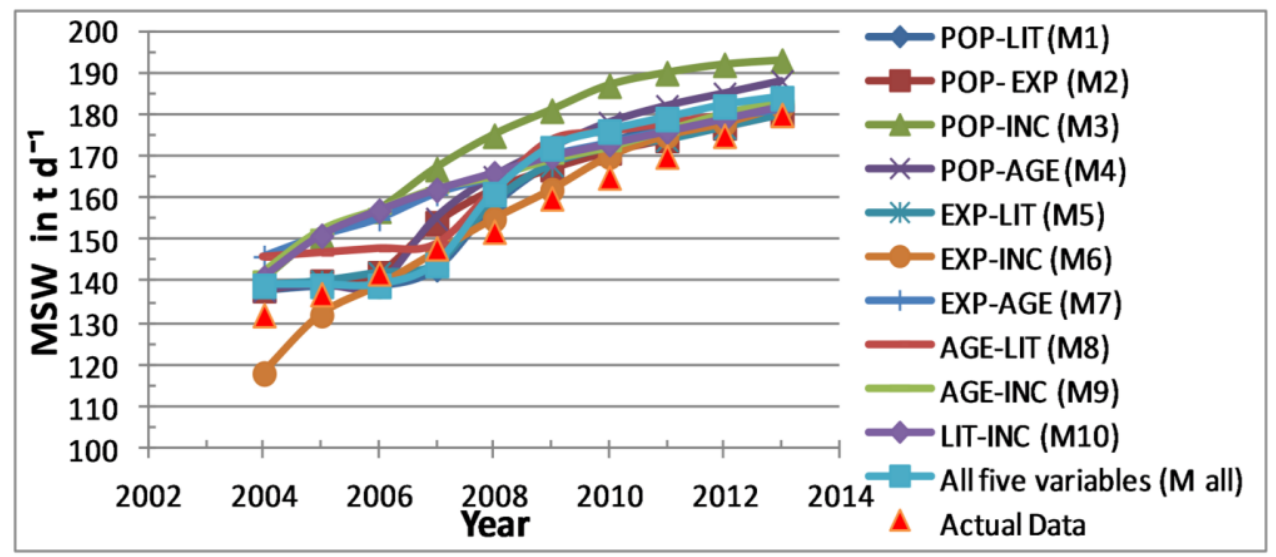

Figure 3. MSW generation obtained from models and actual data for the modeling period (2004 -2013): POP-Total population, AGE- Age group, EXP-Monthly per capita consumer expenditure, LIT-Literacy rate, INC-Per capita annual income, M...- M indicates model name

Table 3. Statistical analysis of models

\begin{tabular}{|c|c|c|c|}
\hline $\begin{array}{l}\text { Model } \\
\text { Name. }\end{array}$ & Inputs considered & $\mathbf{R}^{\mathbf{2}}$ & $\begin{array}{c}\text { Std. error } \\
\text { of estimate }\end{array}$ \\
\hline M1 & Total population and literacy rate (POP-LIT) & 0.941 & 4.689 \\
\hline M2 & Total population and monthly per capita consumer expenditure (POP-EXP) & 0.962 & 3.293 \\
\hline M3 & Total population and per capita annual income (POP-INC) & 0.946 & 4.613 \\
\hline M4 & Total population and age group (POP-AGE) & 0.955 & 4.496 \\
\hline M5 & Monthly per capita consumer expenditure and literacy rate (EXP-LIT) & 0.944 & 4.181 \\
\hline M6 & Monthly per capita consumer expenditure and per capita annual income (EXP-INC) & 0.974 & 3.658 \\
\hline M7 & Monthly per capita consumer expenditure and age group (EXP-AGE) & 0.985 & 1.562 \\
\hline M8 & Age group and literacy rate (AGE-LIT) & 0.924 & 4.472 \\
\hline M9 & Age group and per capita annual income (AGE-INC) & 0.969 & 2.406 \\
\hline $\mathrm{M} 10$ & Literacy rate and per capita annual income (LIT-INC) & 0.955 & 2.931 \\
\hline M all & All five variables in single model ( $\mathrm{M}$ all) & 0.931 & 5.410 \\
\hline
\end{tabular}

$\mathrm{R}^{2}$ - coefficient of determination, POP-Total population, AGE- Age group, EXP-Monthly per capita consumer expenditure, LIT-Literacy rate, INC-Per capita annual income, $\mathrm{M}$...- $\mathrm{M}$ indicates model name

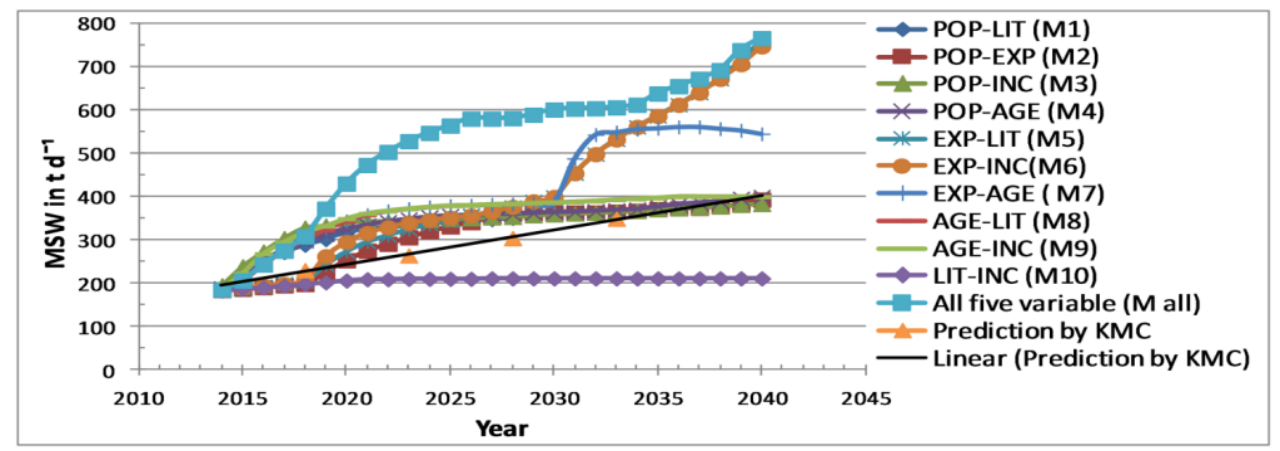

Figure4. MSW generation obtained from models and predicted by KMC for the design period (2014-2040): POP-Total population, AGE- Age group, EXP-Monthly per capita consumer expenditure, LIT-Literacy rate, INC-Per capita annual income, M...- M indicates model name, KMC- Kolhapur Municipal Corporation

From the results, it is clear that monthly per capita consumer expenditure accelerates total waste generation, though many researchers stated that income is the governing attribute for waste generation. High income but low expenditure habit may lead to lesser waste generation. Daskalopoulos et al., (1998) and Zhen-Shan et al., (2011) also identified consumer expenditure as dominating attributes in a waste generation for the resident group. Thanh et al., (2010) revealed a weak correlation between income and household waste generation. Benitez et al., (2008) showed middle-income group generates more waste than higher income. The models having age as input 
variables showed that people having 15 to 59 ages were responsible for more waste generation than the other age groups as shown in the 3D surface view of Figure 5.The predicted results of MSW generation and the data related input variables were also obtained statistically significantly correlated [0.01 level (2-tailed)] for the design period as shown in table 4.

The model considering all the five input variables ( $\mathrm{M}$ all) as shown in Table 3 has also provided good fitness with the actual values of MSW generation and therefore was used to predict MSW generation for KMC area for the design period. Comparative representation of " $\mathrm{M}$ all" model result with KMC prediction has been shown in Figure 6. It is clear that for the design period " $\mathrm{M}$ all" predicted more waste generation than KMC. "M all" model also revealed that the average annual growth rate of MSW generation is 4.99\% for the years 2004 to2040.
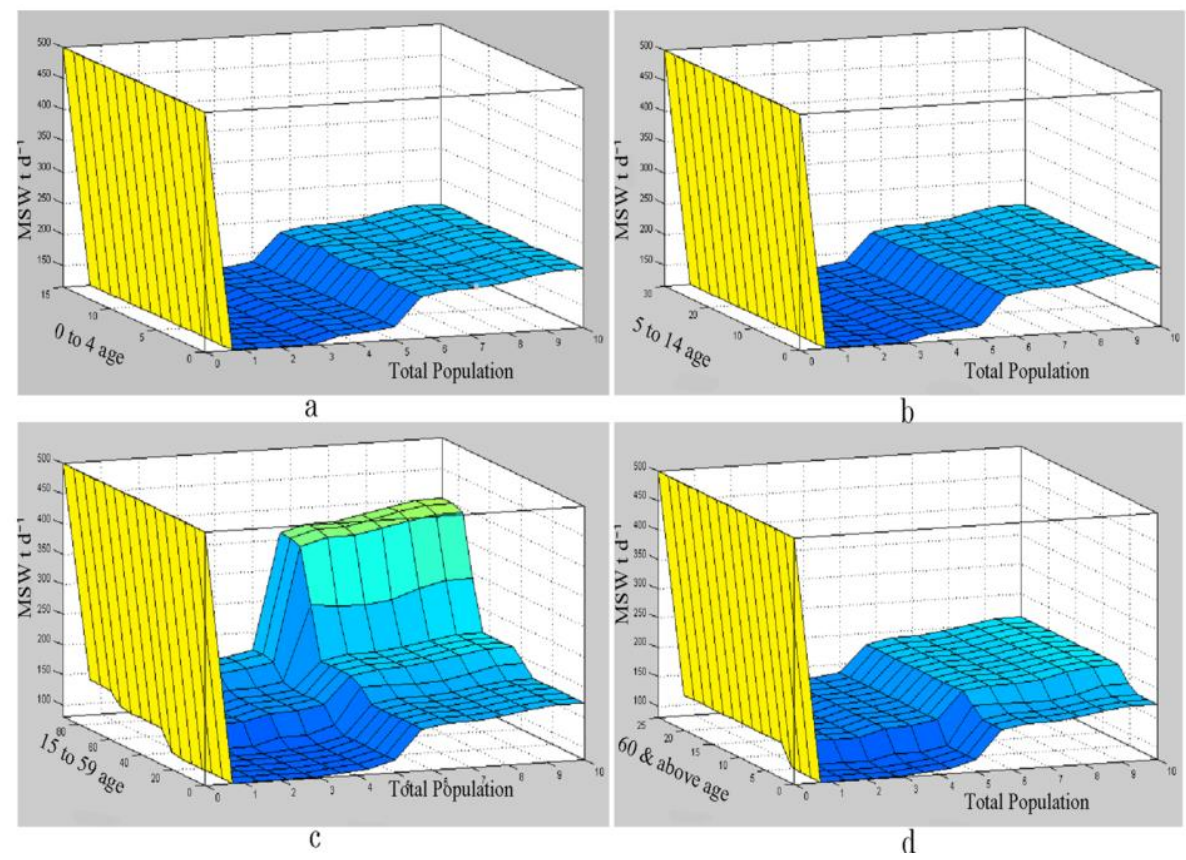

Figure 5. Surface view of age group in \% and total population in Lakh: (a) 0 to 4 age and total population, (b) 5 to 14 age and total population, (c) 15 to 59 age and total population (d) 60 and above age and total population

Table 4. Pearson correlation between variables and output for design period

\begin{tabular}{|c|c|c|c|c|c|c|c|c|c|c|c|c|c|c|c|c|c|c|c|}
\hline & \multirow[b]{2}{*}{ POP } & \multicolumn{4}{|c|}{ AGE } & \multirow[b]{2}{*}{ EXP } & \multirow[b]{2}{*}{ LIT } & \multirow[b]{2}{*}{ INC } & \multirow[b]{2}{*}{ M1 } & \multirow[b]{2}{*}{ M2 } & \multirow[b]{2}{*}{ M3 } & \multirow[b]{2}{*}{ M4 } & \multirow[b]{2}{*}{ M5 } & \multirow[b]{2}{*}{ M6 } & \multirow[b]{2}{*}{ M7 } & \multirow[b]{2}{*}{ M8 } & \multirow[b]{2}{*}{ M9 } & \multirow[b]{2}{*}{ M10 } & \multirow[b]{2}{*}{$M$ all } \\
\hline & & 0 to 4 & 5 to 14 & $\begin{array}{c}15 \text { to } \\
59\end{array}$ & $\begin{array}{c}60 \& \\
\text { above }\end{array}$ & & & & & & & & & & & & & & \\
\hline Pop & 1 & & & & & & & & & & & & & & & & & & \\
\hline 0 to 4 & $-.917^{* *}$ & 1 & & & & & & & & & & & & & & & & & \\
\hline 5 to 14 & $-.998^{* *}$ & $.892^{* *}$ & 1 & & & & & & & & & & & & & & & & \\
\hline Age $\overline{15 \text { to } 59}$ & $.999^{* *}$ & $-.909^{* *}$ & $-.999^{* *}$ & 1 & & & & & & & & & & & & & & & \\
\hline $\begin{array}{c}60 \& \\
\text { above }\end{array}$ & $.998^{* *}$ & $-.893^{* *}$ & $-1.000^{* *}$ & $.999^{* *}$ & 1 & & & & & & & & & & & & & & \\
\hline Exp & $.961^{* *}$ & $-.774^{* *}$ & $-.975^{* *}$ & $.967^{* *}$ & $.975^{* *}$ & 1 & & & & & & & & & & & & & \\
\hline Liter & $999^{* *}$ & $-.933^{* *}$ & $-.995^{* *}$ & $.998^{* *}$ & $.995^{* *}$ & $.950^{* *}$ & 1 & & & & & & & & & & & & \\
\hline Income & $999^{* *}$ & $-.926^{* *}$ & $-.997^{* *}$ & $.999^{* *}$ & $.997^{* *}$ & $.955^{* *}$ & $1.000^{* *}$ & 1 & & & & & & & & & & & \\
\hline M1 & $.877^{* *}$ & $-.967^{* *}$ & $-.848^{* *}$ & $.865^{* *}$ & $.849^{* *}$ & $.742^{* *}$ & $.890^{* *}$ & $.884^{* *}$ & 1 & & & & & & & & & & \\
\hline M2 & $.923^{* *}$ & $-.984^{* *}$ & $-.899^{* *}$ & $.914^{* *}$ & $.899^{* *}$ & $.793^{* *}$ & $.935^{* *}$ & $.929^{* *}$ & $.933^{* *}$ & 1 & & & & & & & & & \\
\hline M3 & $.803^{* *}$ & $-.904^{* *}$ & $-.774^{* *}$ & $.792^{* *}$ & $.775^{* *}$ & $.674^{* *}$ & $.817^{* *}$ & $.810^{* *}$ & $.980^{* *}$ & $.845^{* *}$ & 1 & & & & & & & & \\
\hline M4 & $.866^{* *}$ & $-.954^{* *}$ & $-.838^{* *}$ & $.855^{* *}$ & $.838^{* *}$ & $.737^{* *}$ & $.879^{* *}$ & $.872^{* *}$. & $.996^{* *}$ & $.924^{* *}$ & $.981^{* *}$ & 1 & & & & & & & \\
\hline M5 & $.984^{* *}$ & $-.843^{* *}$ & $-.990^{* *}$ & $.986^{* *}$ & $.990^{* *}$ & $.988^{* *}$ & $.976^{* *}$ & $.980^{* *}$ & $.810^{* *}$ & $.856^{* *}$ & $.739^{* *}$ & $.804^{* *}$ & 1 & & & & & & \\
\hline M6 & $.982^{* *}$ & $-.846^{* *}$ & $-.988^{* *}$ & $.984^{* *}$ & $.988^{* *}$ & $.983^{* *}$ & $.975^{* *}$ & $.978^{* *}$. & $.819^{* *}$ & $.858^{* *}$ & $.750^{* *}$ & $.813^{* *}$ & $.999^{* *}$ & 1 & & & & & \\
\hline M7 & $.951^{* *}$ & $-.908^{* *}$ & $-.946^{* *}$ & $.951^{* *}$ & $.946^{* *}$ & $.891^{* *}$ & $.955^{* *}$ & $.954^{* *}$. & $.888^{* *}$ & $.874^{* *}$ & $.841^{* *}$ & $.873^{* *}$ & $.941^{* *}$ & $.946^{* *}$ & 1 & & & & \\
\hline M8 & $.815^{* *}$ & $-.951^{* *}$ & $-.780^{* *}$ & $.801^{* *}$ & $.780^{* *}$ & $.655^{* *}$ & $.832^{* *}$ & $.823^{* *}$ & $.988^{* *}$ & $.918^{* *}$ & $.971^{* *}$ & $.990^{* *}$ & $.739^{* *}$ & $.751^{* *}$ & $.839^{* *}$ & 1 & & & \\
\hline M9 & $.806^{* *}$ & $-.941^{* *}$ & $-.772^{* *}$ & $.792^{* *}$ & $.772^{* *}$ & $.650^{* *}$ & $.823^{* *}$ & $.815^{* *}$. & $.990^{* *}$ & $.898^{* *}$ & $.985^{* *}$ & $.991^{* *}$ & $.730^{* *}$ & $.742^{* *}$ & $.836^{* *}$ & $.996^{* *}$ & 1 & & \\
\hline M10 & $.756^{* *}$ & $-.925^{* *}$ & $-.715^{* *}$ & $.739^{* *}$ & $.716^{* *}$ & $.578^{* *}$ & $.775^{* *}$ & $.765^{* *}$. & $.964^{* *}$ & $.900^{* *}$ & $.944^{* *}$ & $.967^{* *}$ & $.673^{* *}$ & $.687^{* *}$ & $.780^{* *}$ & $.991^{* *}$ & $.982^{* *}$ & 1 & \\
\hline Mall & $.937^{* *}$ & $-.968^{* *}$ & $-.916^{* *}$ & $.928^{* *}$ & $.916^{* *}$ & $.834^{* *}$ & $.944^{* *}$ & $.940^{* *}$ & $.963^{* *}$ & $.977^{* *}$ & $.903^{* *}$ & $.966^{* *}$ & $.890^{* *}$ & $.895^{* *}$ & $.896^{* *}$ & $.946^{* *}$ & $.932^{* *}$ & $.921^{* *}$ & 1 \\
\hline
\end{tabular}

POP-Total population, AGE- Age group, EXP-Monthly per capita consumer expenditure, LIT-Literacy rate, INC-Per capita annual income, M...- M indicates model name 


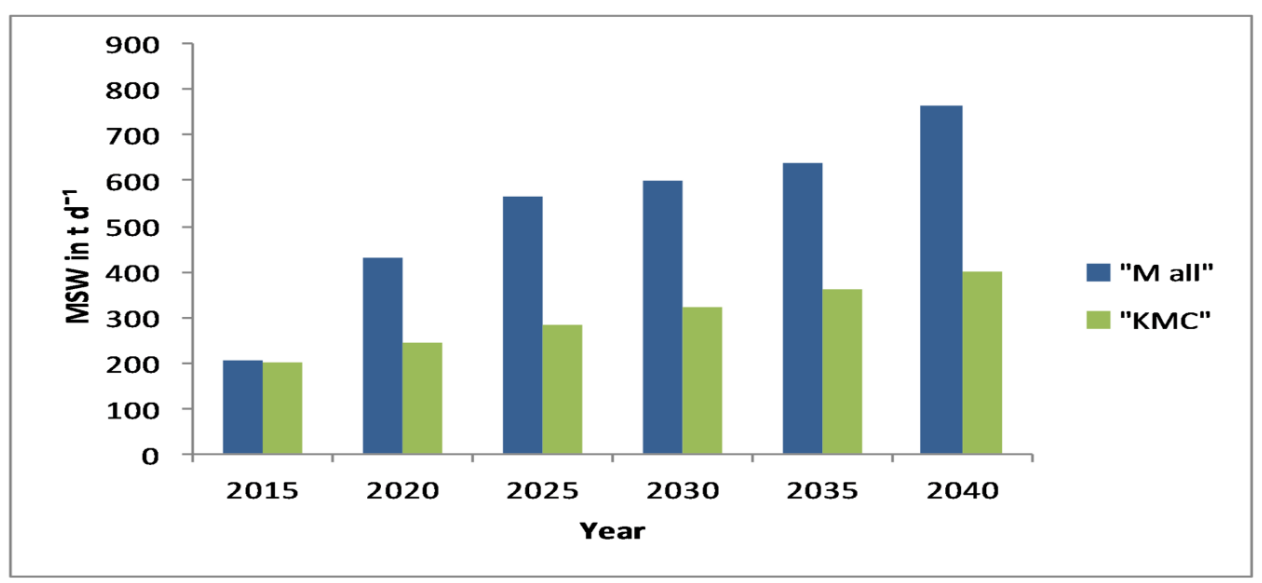

Figure6.MSW generation predicted by the KMC and $\mathrm{M}$ all model for the design period: $\mathrm{M}$ all- All five variables in single model, KMC- Kolhapur Municipal Corporation

\section{Conclusion}

Lack of availability of the continuous data regarding the MSW generation, socio-economic and demographic variables affecting MSW generation makes it difficult to predict MSW generation for the developing countries like India. Conventional models fail to predict MSW generation for aforementioned situations. Moreover, lots of uncertainties are involved with the collected data. Hence, it becomes exceptionally complicated to finalize the most suitable model for waste generation prediction. In this research work, FIS based models were simulated for MSW prediction using local, district and state statistical data which are easily available for the study area. Inputs like total population, per capita annual income, age group, literacy rate and monthly per capita consumer expenditure were considered for predicting the total daily MSW for the design period as well as to study the effect of different input variables on MSW generation. The outputs obtained from the models were validated with the actual waste generation data of KMC area for the modeling period (2004-2013). All valid models were further utilized for future prediction using extended input database for the design period (2014-2040). During validation, it was obtained model with monthly per capita consumer expenditure and age group as input variables is best-fitted since $R^{2}$ value was 0.985 . While in future prediction, models with consumer expenditure as input variable predicted higher waste generation than KMC predicted. Models with population and age as input variables predicted waste generation quite similar as $\mathrm{KMC}^{\prime} \mathrm{s}$ prediction. However, a model with literacy and income as input variables predicted very low waste generation $(211 \mathrm{t}$ $\mathrm{d}^{-1}$ ). While all variables in the single model showed higher waste generation $\left(766 \mathrm{t} \mathrm{d}^{-1}\right.$ ). These results depicted that not only the selection of suitable variables but also developing the model with accurately predicted influencing variables play the important role for MSW generation prediction.

FIS based model using socio-economic and demographic characteristics of the generators were found well-suited models for MSW prediction considering non-uniform period data and hence can be used by the governing bodies to predict accurate MSW generation for designing sustainable MSWM system and selecting suitable landfill site for the design periods. By varying the input data, this technique can be adapted globally since probable all rules were utilized to obtain output result.

\section{Acknowledgements}

The authors are thankful to Kolhapur Municipal Corporation for providing the data regarding MSW generation and different reports that were used for model development and analysis.

\section{References}

Abbasi M., Abduli M.A., Omidvar B. and Baghvand A. (2014), Results uncertainty of support vector machine and hybrid of wavelet transform-support vector machine models for solid waste generation forecasting, Environmental Progress \& Sustainable Energy, 33(1), 220-228.

Arthapedia, 2013, www.arthapedia.in/index.php?title=Cities and Towns. Last accessed 4-3-2017.

Benitez S.O., Lozano-Olvera G., Morelos R.A. and Vega C.A.D. (2008), Mathematical modeling to predict residential solid waste generation, Waste Management, 28, S7-S13.

Daskalopoulos E., Badr O. and Probert S.D. (1998), Municipal solid waste: a prediction methodology for the generation rate and composition in European Union countries and the United States of America, Resources, Conservation and Recycling, 24,155-166.

Directorate of Economics and Statistics, Maharashtra (2012), A report on 'Employment and unemployment situation' based on data collected in state sample of $66^{\text {th }}$ round of national sample survey (July 2009-June 2010), I, 5. www.mahades.maharashtra.gov.in/files/report/nss_66_10_ vol_1.pdf. Last accessed 4-3-2017.

Directorate of Economics and Statistics, Maharashtra (2015), A report on Household Consumer Expenditure based on data collected in state sample of $68^{\text {th }}$ round of national sample survey (July2011-June2012), I, 8. www.mahades.maharashtra.gov.in/files/report/nss_68_1.0 _vol_1_\&_2.pdf. Last accessed 4-3-2017. 
District Socio-Economic Review of Kolhapur (2013), Part 2: 9-11. www.mahades.maharashtra.gov.in/publications.do, Last accessed 4-3-2017.

Gemitzi A., Falalakis G., Eskioglou P. and Petalas C. (2011), Evaluating landslide susceptibility using environmental factors, fuzzy membership function and GIS, Global NEST Journal, 13(1), 28-40.

Gyan research and analytics (2012), Indian income-class opportunities. www.gyananalytics.com/Indian\%20IncomeClass\%200pportunities.pdf, Last accessed 25-7-2017.

Intharathirat R., Salam P.A., Kumar S. and Untong A. (2015), Forecasting of municipal solid waste quantity in a developing country using multivariate grey models, Waste Management, 39, 3-14.

Karadimas N.V., Orsoni A. and Loumous V. (2006), Municipal solid waste generation modelling based on fuzzy logic, Proceedings: $20^{\text {th }}$ European conference on modelling and simulation.

Khan S. and Farooqi I.H. (2012), Prioritising municipal solid waste management factors in India using fuzzy analytic hierarchy process, International Journal Environment and Waste Management, 10(4), 423-440.

Kolekar K.A., Hazra T. and Chakrabarty S.N. (2016), A review on prediction of municipal solid waste generation models, Procedia Environmental Sciences, 35, 238-244.

Kolhapur Municipal Corporation (2008), Detail project report of municipal solid waste management for Kolhapur Municipal Corporation, 68.

Kolhapur Municipal Corporation (2013), Environmental Status Report (ESR) 2012-13, Mass Initiative for Truth Research \& Action (MITRA), 12.

Lozano-Olvera G., Ojeda-Benitez S., Castro-Rodriguez J.R., BravoZanoguera M. and Rodriguez-Diaz A. (2008), Identification of waste packaging profiles using fuzzy logic, Resources, Conservation and Recycling, 52, 1022-1030.

Noori R., Abdoli M.A., Farokhnia A. and Abbasi M. (2009), Results uncertainty of solid waste generation forecasting by hybrid of wavelet transform-ANFIS and wavelet transform-neural network, Expert Systems with Applications, 36, 9991-9999.

Oumarou M.B., Dauda M., Abdulrahim A.T. and Abubakar A.B. (2012), Municipal solid waste generation, recovery and recycling: A case study, World Journal of Engineering and Pure and Applied Science, 2(5), 143-147.

Planning commission of India (2001), Educational development parameters and the preparation of educational development index, Institute of applied manpower research, New Delhi (India), www.planningcommission.nic.in/reports/sereport/ser/stdy_ edudev.pdf, Last accessed 4-3-2017.

Tchobanoglous G., Theisen H. and Vigil S.A. (1993), Integrated solid waste management: engineering principles and management issues, McGraw-Hill, Singapore.

Thanh N.P., Matsui Y. and Fujiwara T. (2010), Household solid waste generation and characteristic in a Mekong Delta city, Vietnam, Journal of Environmental Management, 91, 2307-2321.

Younes M.K., Nopiah Z.M., Basri N.E.A., Basri H., Abushammala M.F.M. and Younes M.Y. (2016), Landfill area estimation based on integrated waste disposal options and solid waste forecasting using modified ANFIS model, Waste Management, 55, 3-11.
Zhen-Shan L., Hui-Zhen F. and Xiao-Yan Q. (2011), Estimating municipal solid waste generation by different activities and various resident groups: A case study of Beijing, Science of the Total Environment, 409, 4406-4414.

Zhen-Shan L., Lei Y., Xiao-Yan Q. and Yu-Mei S. (2009), Municipal solid waste management in Beijing City, Waste Management, 29, 2596-2599.

Zia H. and Devadas V. (2008), Urban solid waste management in Kanpur: Opportunities and perspectives, Habitat International, 32, 58-73. 